\title{
The angiotensin-converting enzyme 2/angiotensin (1-7)/Mas axis protects the function of pancreatic $\beta$ cells by improving the function of islet microvascular endothelial cells
}

\author{
CHUN-LI LU* ${ }^{*}$ YING WANG* ${ }^{*}$, LI YUAN, YANG LI and XIAO-YA LI \\ Department of Endocrinology, Union Hospital, Tongji Medical College, Huazhong University of \\ Science and Technology, Wuhan, Hubei 430022, P.R. China
}

Received February 2, 2014; Accepted July 9, 2014

DOI: $10.3892 /$ ijmm.2014.1917

\begin{abstract}
In the diabetic state, the local rennin-angiotensin system (RAS) is activated in the pancreas, and is strongly associated with islet dysfunction. The angiotensin-converting enzyme 2 (ACE2)/angiotensin (1-7) [Ang(1-7)]/Mas axis is a protective, negative regulator of the classical renin-angiotensin system. In this study, we assessed the role of the ACE2/ Ang(1-7)/Mas axis in pancreatic $\beta$ cell survival and function. ACE2 knockout and wild-type mice were fed a high-fat diet for 16 weeks. We then performed terminal deoxynucleotidyl transferase-mediated dUTP nick end-labeling (TUNEL) assays, and determined the expression levels of interleukin-1 $\beta$ (IL-1 $\beta$ ) and inducible nitric oxide synthase (iNOS) in the pancreatic islets. The effects of Ang(1-7) or Mas receptor silencing on endothelial function were assessed in MS-1 cells. MIN6 cells were then co-cultured with the MS-1 cells to evaluate the effects of ACE2 on insulin secretion. The ACE2 knockout mice were more susceptible than the wild-type mice to high-fat diet-induced $\beta$ cell dysfunction. The TUNEL-positive area of the pancreatic islets and the expression levels of IL-1 $\beta$ and iNOS were markedly increased in the ACE2 knockout mice compared with their wild-type littermates. The Mas-silenced MS-1 cells were more sensitive to palmitate-induced dysfunction and apoptosis in vitro. Ang(1-7) increased the activity of the Akt/endothelial NOS/nitric oxide (NO) pathway in the MS-1 cells, protected MIN6 cells against palmitate-induced apoptosis, and improved MIN6 insulin secretory function in the co-culture system. In conclusion, this study demonstrates that the ACE2/Ang(1-7)/Mas axis is a potential target for protecting the funcion of $\beta$ cells by improving the function of islet microvascular endothelial cells.
\end{abstract}

Correspondence to: Dr Li Yuan, Department of Endocrinology, Union Hospital, Tongji Medical College, Huazhong University of Science and Technology, Wuhan, Hubei 430022, P.R. China E-mail: yuanli18cn@163.com

${ }^{*}$ Contributed equally

Key words: angiotensin (1-7), angiotensin-converting enzyme 2 knockout mice, gene silencing, $\beta$ cells, co-culture

\section{Introduction}

Pancreatic islets in adults are one of the most vascularized organs in the body with a unique, dense, glomerular-like vasculature. The intra-islet capillaries are lined by fenestrated endothelial cells (1). Each $\beta$ cell is surrounded by at least one islet endothelial cell, which may provide signals for islet cell development (2) and may play an important role in adult $\beta$ cell proliferation (3). Endothelial dysfunction is often observed in Asian individuals with insulin resistance and prediabetes (4), and may contribute to the pathogenesis of diabetes mellitus.

The overactivity of the renin-angiotensin system (RAS) in the pancreas is thought to play a pivotal role in the development of diabetes as the structure and function of islets is significantly impaired by local angiotensin II (AngII) (5). We, as well as others, have reported that angiotensin-converting enzyme inhibitors or AngII type 1 receptor blockers attenuate islet damage in diabetic animal models $(5,6)$. We have also reported that improvements in the islet structure and function of type 2 diabetic rats treated with angiotensin-converting enzyme inhibitors or angiotensin receptor blockers are accompanied by significant increases in microvessel density (7). These observations indicate that the blocking of RAS may ameliorate islet function in diabetic rats.

Angiotensin-converting enzyme 2 (ACE2), a homolog of ACE, promotes the degradation of AngII into angiotensin (1-7) [Ang(1-7)] (8,9). Ang(1-7) acts through the G protein-coupled receptor, Mas (10) and opposes many of the actions of AngII (9,11). In diabetic animal models, the loss of ACE2 reverses the negative feedback on RAS, resulting in impaired vascular function or accelerated diabetes-induced kidney injury (12,13). Treatment with Ang(1-7) has been shown to reduce the development of diabetes-induced cardiovascular injury (11). In a previous study using ACE2 knockout mice, we provided indirect evidence that ACE2 plays an important role in improving islet vascularization (14), and this role may be modified after the onset of islet dysfunction.

Nevertheless, the mechanisms through which the ACE2/ Ang(1-7)/Mas axis protects pancreatic islets are not yet fully understood. Therefore, the present study was designed to investigate the roles of ACE2 in pancreatic islets in vivo in mice. We also performed in vitro gain-and loss-of-function experiments to evaluate the precise mechanisms underlying the regulatory 
effects of the ACE2/Ang(1-7)/Mas axis in islet microvascular endothelial cells. Furthermore, since microvascular endothelial cells play an important role in the pathogenesis of $\beta$ cell dysfunction, we investigated the involvement of $\operatorname{Ang}(1-7)$ in the crosstalk between islet microvascular endothelial cells and $\beta$ cells.

\section{Materials and methods}

Animals. Male 5-week-old ACE2 knockout mice and their male wild-type littermates (C57BL/6J) mice as controls were purchased from the Institute of Laboratory Animal Science (Chinese Academy of Medical Sciences, Beijing, China). After 2 weeks of acclimatization, the mice were randomly divided into 2 groups and fed either a regular (control) rodent diet (20\% protein, $70 \%$ carbohydrate and $10 \%$ fat) or a high-fat diet (20\% protein, $20 \%$ carbohydrate and $60 \%$ fat) for 16 weeks. All procedures were approved by the Institutional Animal Care and Use Committee.

Islet immunohistochemistry. Paraffin-embedded pancreatic sections were immunostained for interleukin-1 $\beta$ (IL-1 $\beta$ ) and inducible nitric oxide synthase (iNOS) as previously described (6). Islet cell apoptosis was determined by terminal deoxynucleotidyl transferase dUTP nick end-labeling (TUNEL) staining.

Cell culture. Palmitate, bovine serum albumin (BSA) and Krebs-Ringer bicarbonate HEPES buffer (KRBH) were purchased from Sigma (St. Louis, MO, USA). Islet microvascular endothelial cells (MS-1) were purchased from the Chinese Academy of Sciences Cell Bank, Shanghai, China. These cells were cultured in basal medium consisting of Dulbecco's modified Eagle's medium (DMEM) (HyClone/Thermo Scientific, Waltham, MA, USA). Pancreatic MIN6 $\beta$ cells (passages 35-45) were a gift from Professor Hui Zhou (Tongji Hospital, Tongji Medical College, Huazhong University of Science and Technology, Hubei, China), and were maintained in DMEM containing $25 \mathrm{mmol} / 1$ glucose. The culture media for both cell lines were supplemented with 15\% fetal bovine serum (Gibco/ Life Technologies, Carlsbad, CA, USA), $100 \mathrm{U} / \mathrm{ml}$ penicillin and $100 \mu \mathrm{g} / \mathrm{ml}$ streptomycin under standard conditions (5\% $\mathrm{CO}_{2}$ and $\left.37^{\circ} \mathrm{C}\right)$.

Gene silencing. MS-1 cells were plated in 6-well dishes. The cells were transfected with small-interfering RNA (siRNA) specific for the Mas receptor for $6 \mathrm{~h}$ using Lipofectamine 2000 (Invitrogen, Carlsbad, CA, USA) in serum-free culture medium. Subsequently, the transfection medium was replaced with full culture medium, as described in the manufacturer's instructions. The control cells were exposed to the transfectant in the absence of siRNA. The target sequences for the mouse Mas receptor were 5'-GUCCUCUACUUGCUGUACUTT-3' and 5'-AGUACAGCAAGUAGAGGACTT-3'.

NO assay. The MS-1 cells were stimulated with Ang(1-7) $\left(10^{-9}-10^{-5} \mathrm{~mol} / \mathrm{l}\right)$ for $10 \mathrm{~min}$ with or without $\mathrm{A} 779$, a potent and selective Ang(1-7) antagonist (10-6 $\mathrm{mol} / \mathrm{l})$ (GL Biochem, Shanghai, China). In some experiments, the cells were preexposed to an inhibitor of NOS, $\mathrm{N}^{\mathrm{G}}$-nitro-L-arginine methyl ester (L-NAME) $\left(10^{-5} \mathrm{~mol} / 1 ; 30 \mathrm{~min}\right)$ (Beyotime Institute of
Biotechnology, Shanghai, China). The NO concentration in the cell supernatant was determined using an $\mathrm{NO}$ assay according to the instructions of the manufacturer (Nanjing Jiancheng Bioengineering Institute, Nanjing, China).

Western blot analysis. The MS-1 cells were stimulated with Ang(1-7) $\left(10^{-6} \mathrm{~mol} / \mathrm{l}\right)$ for $10 \mathrm{~min}$ with or without A779. In some experiments, the cells were pre-exposed to the Akt inhibitor, wortmannin $\left(10^{-6} \mathrm{~mol} / 1\right.$; $\left.30 \mathrm{~min}\right)$ (Sigma). The cells were harvested and lysed in cell lysis buffer (Beyotime Institute of Biotechnology). Protein samples $(30 \mu \mathrm{g})$ were separated by $8 \%$ sodium dodecyl sulfate-polyacrylamide gel electrophoresis and transferred onto pure nitrocellulose membranes. The membranes were blocked and incubated with one of the specific antibodies overnight at $4^{\circ} \mathrm{C}$. The membranes were then probed with peroxidase-conjugated goat anti-mouse IgG (1:5,000; Pierce Biochemicals/Thermo Scientific) or peroxidase-conjugated goat anti-rabbit IgG (1:5,000; Amersham PLC, Amersham, UK) for $1 \mathrm{~h}$ at room temperature, which was followed by chemiluminescence detection (Amersham PLC). The following antibodies were used in this study: anti-phosphorylated (p)-Akt (Ser473), anti-p-endothelial NO synthase (eNOS) (Ser1177), anti-Akt (1:1,000; Cell Signaling Technology, Beverly, MA, USA), anti-eNOS, anti-caspase-3, anti-Bax and anti-Bcl-2 (1:1,000; Santa Cruz Biotechnology, Inc., Santa Cruz, CA, USA).

Real-time polymerase chain reaction (PCR). Total RNA was extracted using TRIzol reagent (Takara, Tokyo, Japan) according to the manufacturer's instructions. Total RNA $(500 \mathrm{ng})$ was used for cDNA synthesis using a cDNA reverse transcription kit (Takara. PCR was carried out in 96-well plates using the ABI Prism 7900HT Sequence Detection System (Applied Biosystems, Bedford, MA, USA). The primer sequences were as follows: Mas, 5'-GCTTGAGGCTATCTTCCTGTGATC-3' (forward) and 5'-GAGGCTTCCAAACTCAGTCAGTC-3' (reverse); and GAPDH,5'-GGTGAAGGTCGGTGTGAACG-3' (forward) and 5'-CTCGCTCCTGGAAGATGGTG-3' (reverse).

MIN6 and MS-1 cell co-culture. The MIN6 and MS-1 cells were co-cultured in 6-well plates containing high pore density and polyethylene terephthalate track-etched cell culture inserts (0.4 $\mu \mathrm{m}$ pores) (BD Biosciences Labware Europe, Le Pont De Claix, France). The MS-1 cells $(50,000$ cells/well) were pre-treated with or without $\operatorname{Ang}(1-7)\left(10^{-6} \mathrm{~mol} / \mathrm{l}\right)$ for $24 \mathrm{~h}$, and were plated onto the bottom surface of each well. The MIN6 cells $(25,000$ cells/well), which were seeded onto the inner membrane of the cell culture insert, were pre-treated with $0.5 \%$ (wt/vol) BSA or with $0.5 \mathrm{mmol} / 1$ palmitate in BSA for $24 \mathrm{~h}$, and were then placed in the well. Co-culture continued for $24 \mathrm{~h}$. As a control, the MIN6 cells were cultured in the cell culture insert in the absence of MS-1 cells.

Glucose-stimulated insulin secretion (GSIS). The MIN6 cells were washed with phosphate-buffered saline twice and were then pre-incubated in $\mathrm{KRBH}$ and $2.8 \mathrm{mmol} / 1$ glucose for $60 \mathrm{~min}$ at $37^{\circ} \mathrm{C}$. GSIS was then assessed by serial incubations for $60 \mathrm{~min}$ in KRBH containing 2.8 or $16.7 \mathrm{mmol} / \mathrm{l}$ glucose. At the end of the incubation period, the insulin concentration in the supernatant was measured using an assay kit (Millipore, Billerica, MA, USA). 

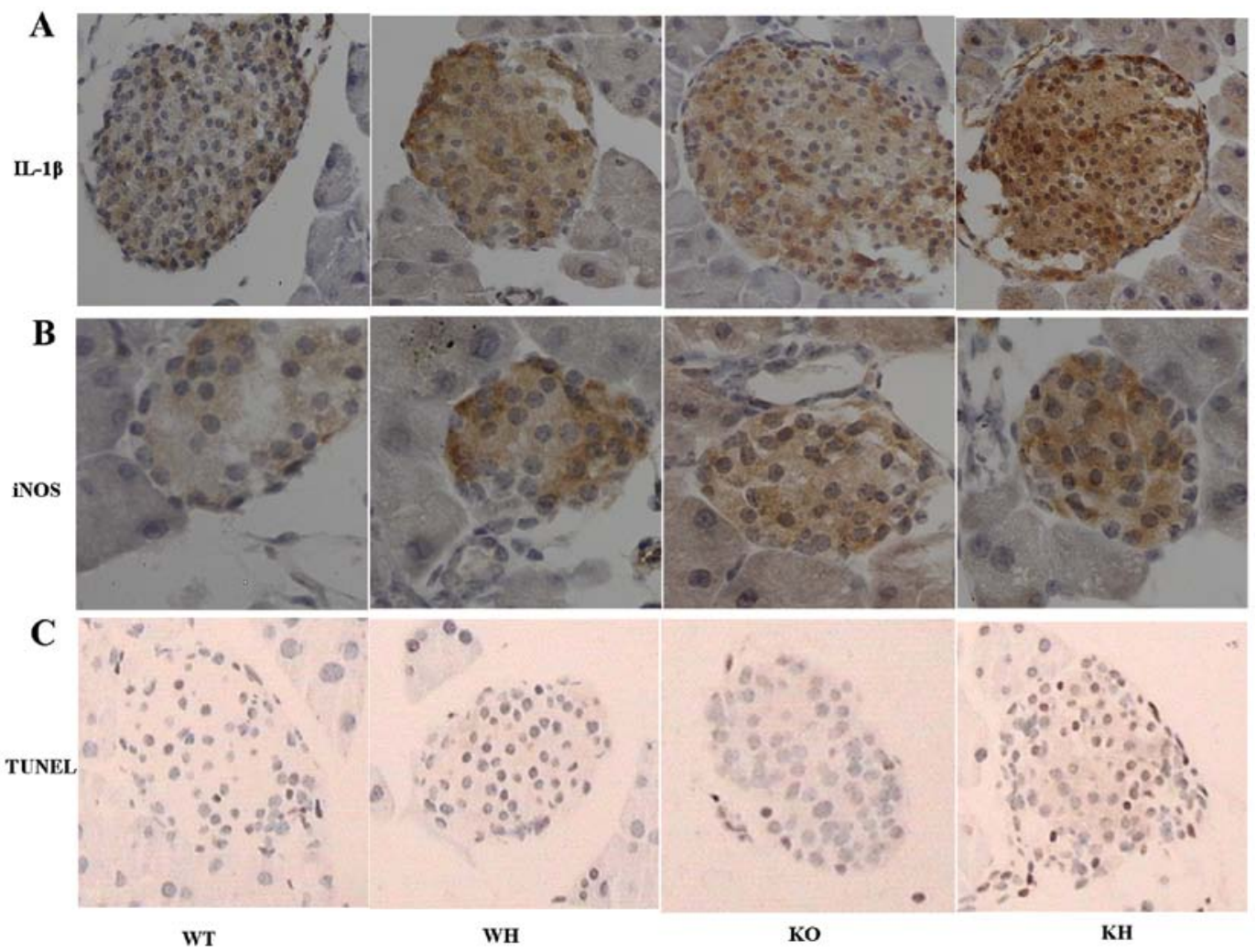

Figure 1. (A and B) Immunohistochemical staining for (A) pancreatic interleukin-1 $\beta$ (IL-1ß) and (B) inducible nitric oxide synthase (iNOS) protein expression. (C) Terminal deoxynucleotidyl transferase-mediated dUTP nick end-labeling (TUNEL) staining of the pancreas. Magnification, $x 400$. WT, wild-type mice fed regulat diet; WH, wild-type mice fed the high-fat diet; KO, angiotensin-converting enzyme 2 (ACE2) knockout mice fed regular diet; KH, ACE2 knockout mice fed the high-fat diet.

Analysis of cell apoptosis. After the indicated treatments, the cells were harvested and the rate of apoptosis was determined by flow cytometry using an Annexin V-FITC/PI assay (BD Pharmingen, Franklin Lakes, NJ, USA) according to the manufacturer's instructions.

Statistical analysis. All data are presented as the means \pm standard error of at least 3 independent experiments. The significance of differences among the experimental groups was determined by analysis of variance. Values of $\mathrm{P}<0.05$ were considered to indicate statistically significant differences. Statistical analyses were performed using GraphPad Prism software version 5.0 (GraphPad Software Inc., San Diego, CA, USA).

\section{Results}

ACE2 knockout mice are more susceptible than wild-type mice to high-fat diet-induced $\beta$ cell apoptosis. ACE2 deficiency did not significantly affect $\beta$ cell survival, although the TUNEL-positive area in the islets was slightly larger in the ACE2 knockout mice than in the wild-type mice fed the control diet (Fig. 1). However, in the mice fed the high-fat diet, the TUNEL-positive area in the islets was much larger in the ACE2 knockout mice than in the wild-type mice, which suggests that theACE2 knockout mice are more susceptible to high-fat diet-induced $\beta$ cell apoptosis.

The expression of IL-1 $\beta$ and iNOS was not affected in the ACE2 knockout mice fed the control diet. However, after being fed the high-fat diet for 16 weeks, the expression levels of both IL-1 $\beta$ and iNOS were increased in the ACE2 knockout mice compared to the wild-type mice.

Blocking the ACE2/Ang(1-7)/Mas axis aggravates palmitateinduced MS-1 cell dysfunction. Mas receptors are constitutively expressed in endothelial cells, providing a physiological basis for the effects of Ang(1-7) in endothelial cells (15). The endothelium is a key target of activated ACE2 (16). To characterize the role of the ACE2/Ang(1-7)/Mas axis in islet microvascular endothelial cells, we used siRNA to silence the Mas receptor, and thus block the ACE2/Ang(1-7)/Mas axis in the MS-1 cells. The transfection efficacy was confirmed by real-time PCR (Fig. 2A). The phosphorylation of eNOS at Ser1177 in the siRNA-treated MS-1 cells did not differ significantly from that of the control MS-1 cells. However, exposure to palmitate for $24 \mathrm{~h}$ significantly decreased eNOS phosphorylation in the siRNA-treated MS-1 cells compared with the MS-1 cells $(\mathrm{P}<0.05$; Fig. 2B). Consistent with these findings, NO levels was significantly decreased in the MS-1 cells exposed to palmitate, and was further reduced in the cells treated with Mas siRNA (Fig. 2C).

We then examined the activation of the upstream phosphatidylinositide 3-kinase (PI3K)/Akt signaling pathway. Akt phosphorylation in the MS-1 cells was reduced by Mas siRNA, albeit not significantly compared with the control cells. Exposure to palmitate for $24 \mathrm{~h}$ significantly reduced Akt phosphorylation in the MS-1 cells treated with Mas siRNA by $61.9 \%$ relative to the MS-1 cells treated with palmitate alone $(\mathrm{P}<0.05)$. Taken together, these data confirmed that the 

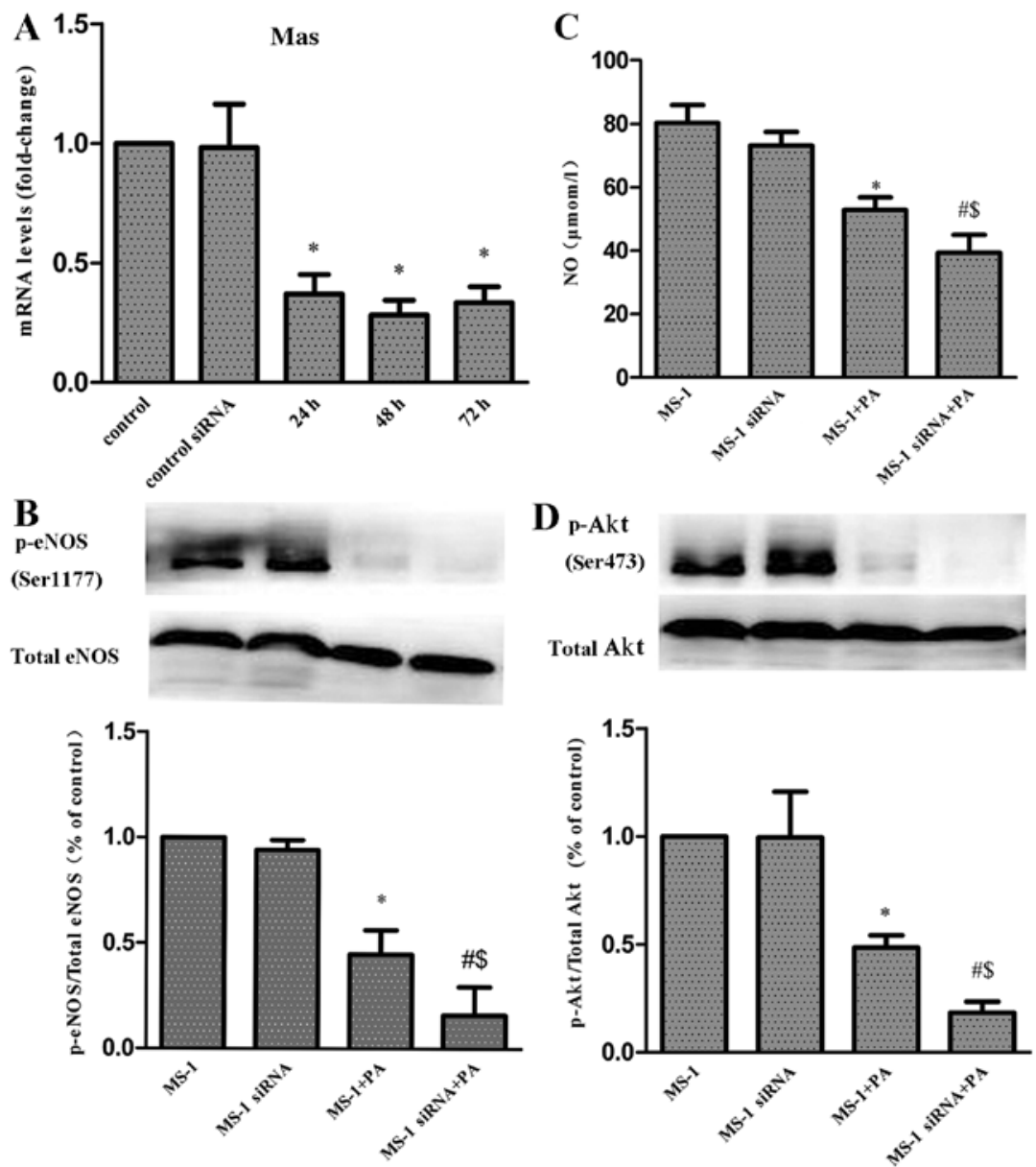

Figure 2. Transfection with small-interfering RNA (siRNA) targeting Mas enhances the palmitate-induced endothelial dysfunction of MS-1 cells. (A) Mas mRNA levels following transfection with siRNA for Mas. ${ }^{*} \mathrm{P}<0.05$ vs. control siRNA. (B and D) Representative western blots showing the protein expression levels of (B) endothelial nitric oxide synthase (eNOS) and (D) Akt. (C) Culture media nitric oxide (NO) levels. Values are the means \pm standard error ( $\mathrm{n}=5$ ). ${ }^{*} \mathrm{P}<0.05$ vs. untreated MS-1 cells (MS-1); ${ }^{*} \mathrm{P}<0.05$ vs. siRNA-treated MS-1 cells (MS-1 + siRNA); ${ }^{\circ} \mathrm{P}<0.05$ vs. palmitate-treated MS- 1 cells (MS-1 + PA).

palmitate-induced endothelial dysfunction was aggravated by Mas silencing in the MS-1 cells (Fig. 2D).

Blocking the ACE2/Ang(1-7)/Mas axis aggravates palmitateinduced apoptosis of $M S-1$ cells. The $\mathrm{Bax} / \mathrm{Bcl}-2$ ratio in the Mas siRNA-treated MS-1 cells did not differ significantly from that in the control MS-1 cells (Fig. 3A). However, exposure to palmitate for $24 \mathrm{~h}$ increased the $\mathrm{Bax} / \mathrm{Bcl}-2$ ratio by $43.4 \%$ in the siRNA-treated MS-1 cells compared with the MS-1 cells treated with palmitate alone $(\mathrm{P}<0.05)$. The cleavage of caspase-3 (17 kDa) was also increased by $48.8 \%$ in the MS-1 cells treated with Mas siRNA and palmitate $(\mathrm{P}<0.05)$ (Fig. 3B), which was indicative of severe apoptosis in the Mas-silenced cells.

Ang(1-7) activates the Akt/eNOS/NO pathway through Mas in MS-1 cells. In order to ascertain whether the activation of the ACE2/Ang(1-7)/Mas signaling pathway improves endothelial function, the MS-1 cells were stimulated with Ang(1-7) and the activity of the Akt/eNOS/NO pathway was then determined. We found that Ang(1-7) significantly increased eNOS phosphorylation at Ser1177 in a time-dependent manner; this effect was blocked by A779 (Fig. 4A). Wortmannin (a PI3K inhibitor; $\left.10^{-6} \mathrm{~mol} / \mathrm{l} ; 30 \mathrm{~min}\right)$ suppressed these effects of
Ang(1-7) on eNOS phosphorylation (Fig. 4B), indicating that Ang(1-7) induces eNOS phosphorylation through the PI3K/ Akt signaling pathway.

Ang(1-7) significantly enhanced NO production by MS-1 cells, which was blocked by A779 (Fig. 4D). L-NAME, an inhibitor of NOS, also inhibited the Ang(1-7)-induced NO generation. These results indicate that Ang(1-7) stimulates NO formation through the Mas receptor, which is coupled to eNOS.

We also observed that $\operatorname{Ang}(1-7)$ at the same dose induced Akt phosphorylation at Ser473 in a time-dependent manner, reaching a maximum level at $10 \mathrm{~min}$. A779 blocked the effects of Ang(1-7) on Akt phosphorylation (Fig. 4C).

Ang(1-7) restores insulin secretion in palmitate-treated MIN6 cells co-cultured with MS-1 cells. To determine whether the effects of $\operatorname{Ang}(1-7)$ on MS-1 cells alter the function of MIN6 cells, the MIN6 cells pre-incubated with palmitate were co-cultured with the MS-1 cells, and the insulin secretory capacity was assessed. Palmitate increased basal insulin secretion, but decreased GSIS in the MIN6 cells (both $\mathrm{P}<0.05$ ) (Fig. 5). GSIS was partly restored by co-culture with MS-1 cells. Although basal insulin secretion was not affected when the MIN6 cells were co-cultured with Ang(1-7)-treated MS-1 cells, 

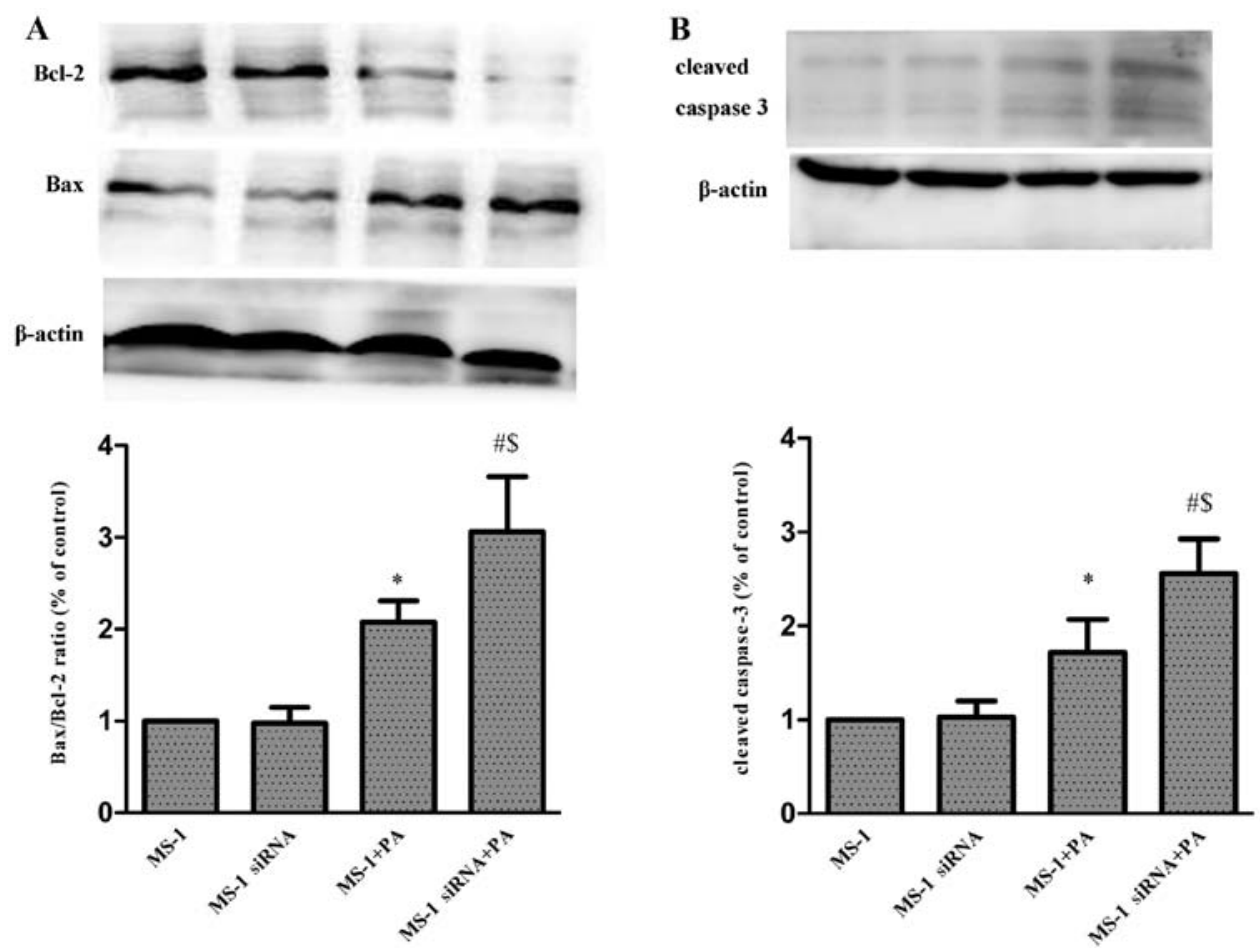

Figure 3. Transfection with small-interfering RNA (siRNA) targeting Mas enhances the palmitate-induced endothelial apoptosis of MS-1 cells (A and B) Representative western blots showing the protein expression levels of (A) Bax/Bcl-2 and (B) caspase-3. Values are the means \pm standard error ( $\mathrm{n}=5$ ). ${ }^{\prime \prime} \mathrm{P}<0.05$ vs. untreated MS-1 cells (MS-1); ${ }^{\text {P }}<0.05$ vs. siRNA-treated MS-1 cells (MS- $1+$ siRNA); ${ }^{~} \mathrm{P}<0.05$ vs. palmitate-treated MS- 1 cells (MS-1 + PA).

GSIS was increased by 1.15 -fold in response to $16.7 \mathrm{mmol} / \mathrm{l}$ glucose $(\mathrm{P}<0.05)$ compared with that in MIN6 cells co-cultured with untreated MS-1 cells. This effect of Ang(1-7) was blocked by pre-incubating the MS-1 cells with A779. These results indicate that co-culture with Ang(1-7)-treated MS-1 cells improved GSIS in palmitate-treated MIN6 cells.

Ang(1-7) attenuates the palmitate-induced apoptosis of MIN6 cells co-cultured with MS-1 cells. As shown in Fig. 6, palmitate increased the apoptotic rate of the MIN6 cells by approximately 5.6-fold compared with the control MIN6 cells treated with BSA only (Fig. 6A vs. B; $\mathrm{P}<0.05$ ). Of note, co-culture with the MS-1 cells decreased the apoptotic rate of the palmitate-treated MIN6 cells by $37.3 \%$ (Fig. 6C). Co-culture with the Ang(1-7)treated MS-1 cells decreased the palmitate-induced apoptotic rate of the MIN6 cells by $65.1 \%$ (Fig. 6D); this reduction was significantly greater than the reduction observed in the MIN6 cells co-cultured with untreated MS-1 cells $(\mathrm{P}<0.05)$. This antiapoptotic effect of Ang(1-7) was blunted by A779 (Fig. 6E). These results indicate that $\mathrm{Ang}(1-7)$ enhances the protective effects of MS-1 cells on the palmitate-induced apoptosis of MIN6 cells.

\section{Discussion}

The current study provides direct evidence that the ACE2/ Ang(1-7)/Mas axis protects $\beta$ cells from apoptosis and improves their function. The absence of ACE2 in mice enhances the longterm high-fat diet-induced expression of IL-1 $\beta$ and iNOS in pancreatic islets. These effects may be related to $\beta$ cell death and possibly involve islet microvascular endothelial cell dysfunction. This principle was further confirmed by the co-culture system in which Ang(1-7) improved endothelial function and protected the MIN6 cells against palmitate-induced dysfunction and apoptosis. These data suggest that the ACE2/Ang(1-7)/Mas axis maintains islet microvascular endothelial homeostasis, and plays a permissive role in the survival and function of islet $\beta$ cells.

ACE2 cleaves the terminal phenylalanine residue from AngII to generate Ang(1-7), and thus decreases AngII levels (8,9). Ang(1-7) exerts protective effects by enhancing vasodilatation and NO generation, which counteract many of the activities of AngII $(11,16)$. Diabetes is characterized by the activation of local RAS in the pancreas, which affects $\beta$ cell function by increasing inflammation, oxidative stress, intraislet fibrosis and apoptosis $(5,6)$. ACE2 protein and/or mRNA expression levels have been shown to be increased in young Zucker diabetic fatty rats (5) and in young $d b / d b$ mice (17). These changes may represent an adaptive compensation to combat the increasing levels of ACE and AngII. In our previous study, we demonstrated that a high-fat diet caused progressive glucose intolerance in ACE2 knockout mice (14). In the present study, we observed marked increases in the apoptosis and expression of IL-1 $\beta$ and iNOS in the pancreatic islets of ACE2 knockout mice fed a high-fat diet. Conversely, pancreatic ACE2 gene therapy has been shown to improve glycemic control in diabetic $d b / d b$ mice by improving $\beta$ cell function (17). The loss of the counter regulatory activity of the ACE2/Ang(1-7)/Mas axis may contribute to $\beta$ cell dysfunction.

In our previous studies, RAS blockade improved islet function and reduced apoptosis in parallel with an increased density of microvessels in the islets $(6,18)$. Consistent with these findings, long-term high-fat dietary feeding induced a greater decrease in islet capillary density in ACE2 knockout 
$\mathbf{A}$
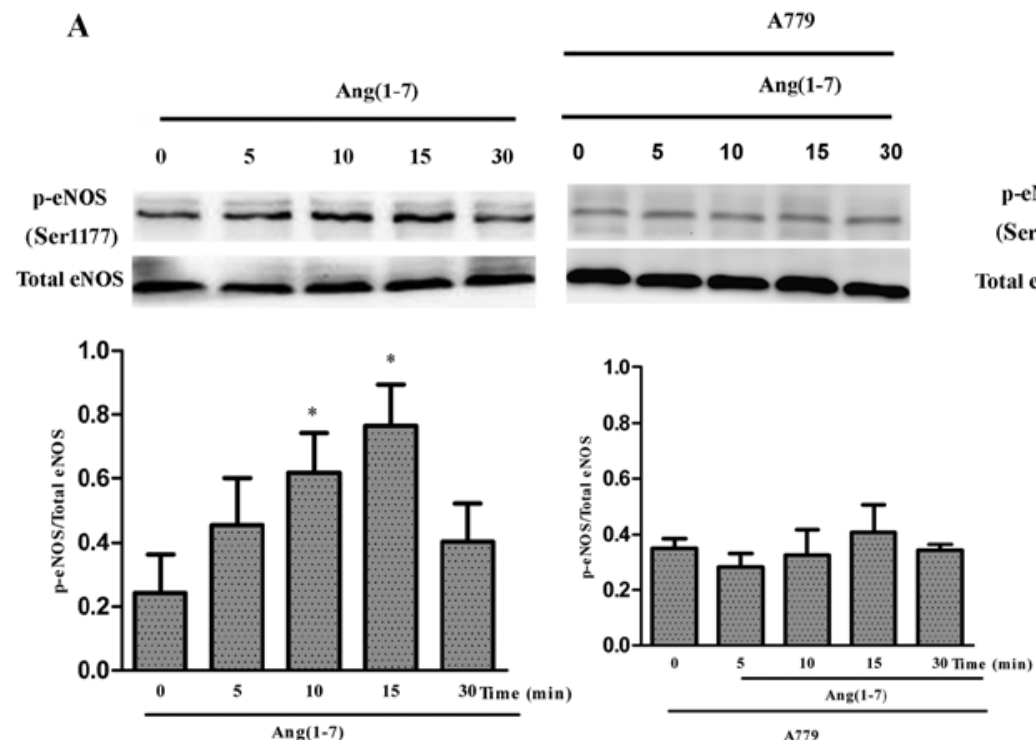

C
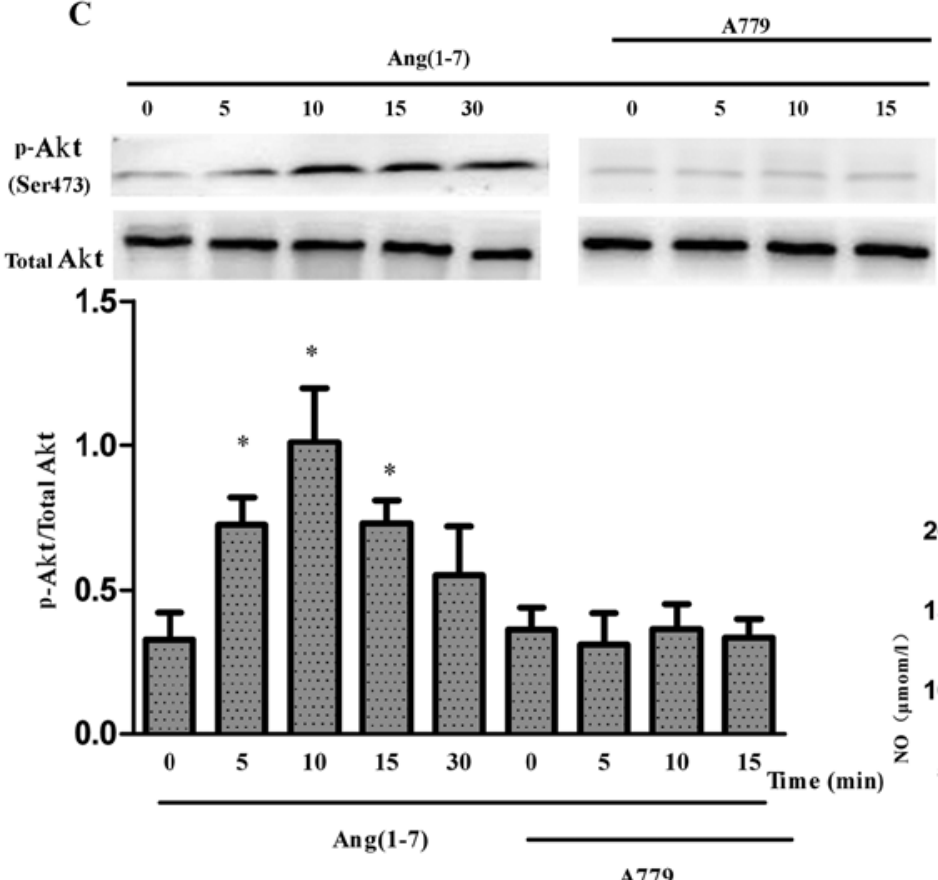

B

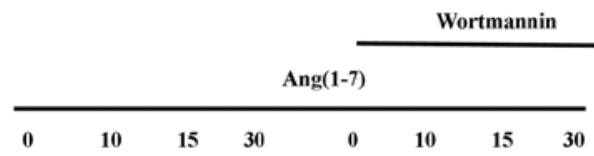

p-eNOS

(Ser1177)

Total eNos

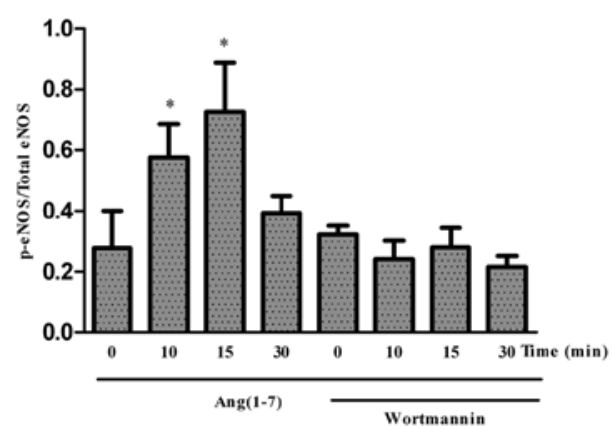

D

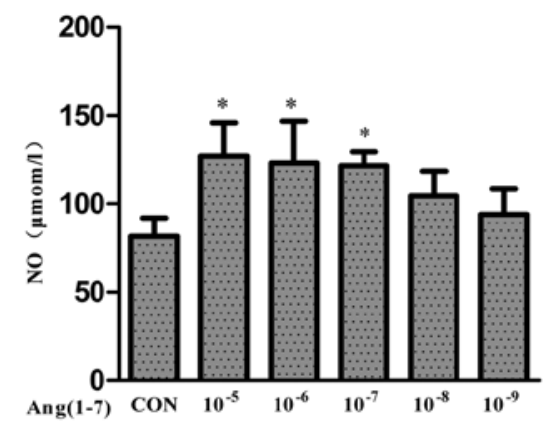

Figure 4. Angiotensin (1-7) [Ang(1-7)] increases the activity of the Akt/endothelial nitric oxide synthase (eNOS)/NO signaling pathway in MS-1 cells. MS-1 cells were treated with Ang(1-7) (10-6 $\mathrm{mol} / \mathrm{l})$ without or with A779 [Ang(1-7) antagonist; $\left.10^{-6} \mathrm{~mol} / 1\right]$ or wortmannin (PI3K inhibitor). Representative western blots showing the protein expression levels of (A and B) eNOS and (C) Akt. Values are the means \pm standard error (SE) ( $\mathrm{n}=3$ ). ${ }^{*} \mathrm{P}<0.05$ vs. 0 min. (D) MS-1 cells were treated with Ang (1-7) $\left(10^{-9}-10^{-5} \mathrm{~mol} / \mathrm{l}\right)$ for $30 \mathrm{~min}$ without or with A779 $\left(10^{-6} \mathrm{~mol} / \mathrm{l}\right)$ or $\mathrm{N}^{\mathrm{G}}$-nitro-L-arginine methyl ester (L-NAME), and the NO levels in the cell supernatants were measured. Values are the means $\pm \mathrm{SE}(\mathrm{n}=3)$. ${ }^{*} \mathrm{P}<0.05$ vs. control cells.

mice (14). The reduced capillary density in ACE2 knockout mice may not be limited to islets, as a similar phenomenon was detected in the kidneys of these mice (19). Conversely, treatment with Ang(1-7) improved the pancreatic microcirculation and attenuated $\beta$ cell dysfunction (20). These results indicate that ACE2 may prevent AngII-induced microvessel dysfunction.

The intra-islet capillaries are lined with fenestrated endothelial cells (1). Circulatory and paracrine signals derived from endothelial cells are essential for inducing pancreatic develop- ment, endocrine cell differentiation and the maintenance of islet function (21). An earlier study suggested that the AngII type 1 receptor signaling pathway in endothelial cells induces endothelial dysfunction, possibly by inhibiting NO (22). The effects of AngII in kidney microvessels are also greatly reduced by NO (23). The current study demonstrated that the abolishment of the ACE2/Ang(1-7)/Mas axis by the genetic silencing of Mas enhanced the palmitate-induced activity of the Akt/eNOS/NO pathway. Conversely, Ang(1-7) increased NO synthesis and the phosphorylation of both Akt and eNOS. We also found that 


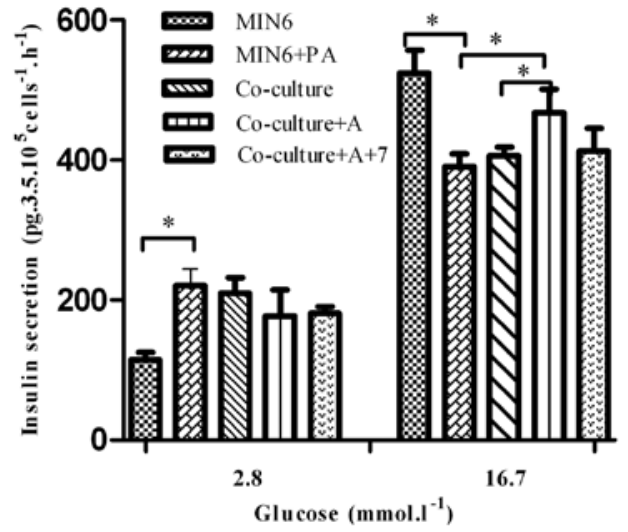

Figure 5. Angiotensin (1-7) [Ang(1-7)] inhibits palmitate-induced MIN6 cell dysfunction during co-culture with MS-1 cells. MIN6 cells were treated for $24 \mathrm{~h}$ and glucose-stimulated insulin secretion (GSIS) was measured. Palmitate increased basal insulin secretion, but decreased GSIS in the MIN6 cells. Values are the means \pm standard error $(n=5)$. "P<0.05. MIN6 group, MIN6 cells were exposed to $0.5 \%$ bovine serum albumin (BSA); MIN6 + PA group, MIN6 cells were exposed to $0.5 \mathrm{mmol} / 1$ palmitate plus $0.5 \% \mathrm{BSA}$; coculture group, MIN6 cells were exposed to $0.5 \mathrm{mmol} / 1$ palmitate plus $0.5 \%$ BSA, and were co-cultured with untreated MS-1 cells; co-culture + A group, MIN6 cells were exposed to $0.5 \mathrm{mmol} / 1$ palmitate plus $0.5 \% \mathrm{BSA}$, and were co-cultured with MS-1 cells pre-treated with $10^{-6} \mathrm{~mol} / \mathrm{l} \mathrm{Ang}(1-7)$; coculture $+\mathrm{A}+7$ group, MIN6 cells were exposed to $0.5 \mathrm{mmol} / \mathrm{l}$ palmitate plus $0.5 \% \mathrm{BSA}$, and were co-cultured with MS-1 cells pre-treated with $10^{-6} \mathrm{~mol} / 1$ Ang(1-7) and $10^{-6} \mathrm{~mol} / \mathrm{l} \mathrm{A779}$. palmitate-induced apoptosis was increased by Mas silencing in MS- 1 cells with the activation of the Bcl-2 and caspase- 3 pathways. Our previous in vivo study demonstrated that ACE2 deficiency aggravated high-fat diet-induced insulin secretory dysfunction and greatly decreased islet microvascular vessel density (14). Taken together, these data suggest that the activation of the ACE2/Ang(1-7)/Mas axis protects $\beta$ cells against dysfunction and apoptosis by enhancing microvascular endothelial cell survival and function.

We performed in vitro studies using a co-culture system to confirm our in vivo findings. Song et al (24) reported that islet function was improved when rat islets were co-cultured with endothelial cells. Of note, we found that MIN6 cells displayed marked improvements in glucose tolerance when they were co-cultured with MS-1 cells treated with Ang(1-7) compared with MIN6 cells co-cultured with untreated MS-1 cells, and this effect was attenuated by A779. Increased vascularization of the pancreas not only improved secretory function, but may also reduce islet apoptosis in mice (25). We previously reported that the loss of ACE 2 decreased the number of $\beta$ cells in high-fat diet-fed mice (14). The present results obtained using a co-culture system revealed that Ang(1-7) attenuated the palmitate-induced apoptosis of MIN6 cells. Therefore, these findings suggest that $\mathrm{Ang}(1-7)$ helps to sense the environ-
A

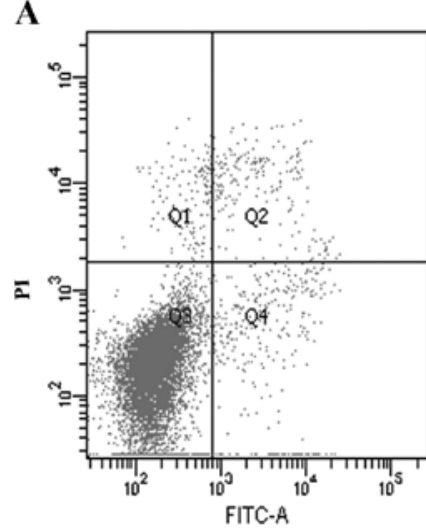

D

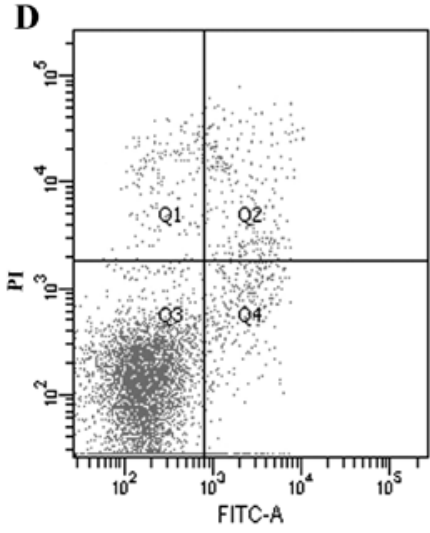

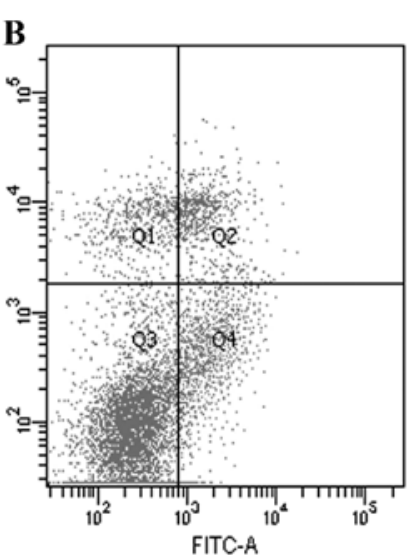

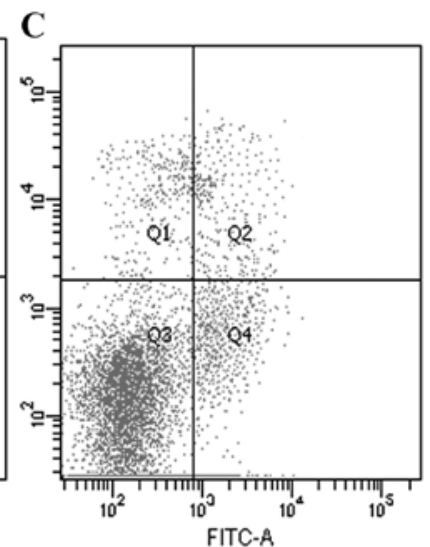

$\mathbf{E}$

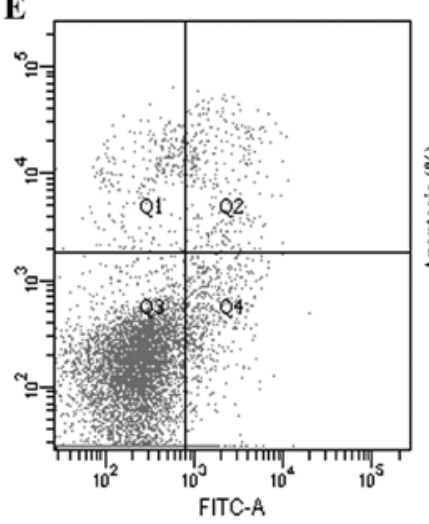

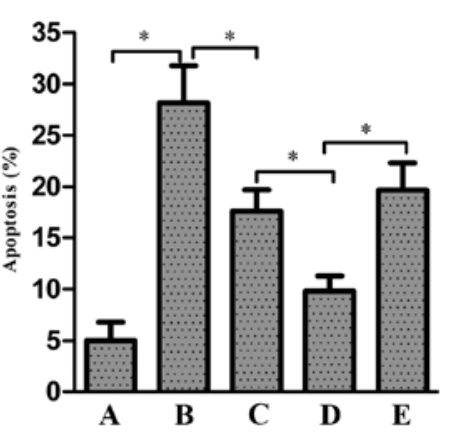

Figure 6. Angiotensin (1-7) [Ang(1-7)] inhibits palmitate-induced MIN6 cell apoptosis during co-culture with MS-1 cells. MIN6 cells were treated for 24 h with the indicated treatments and cell apoptosis was measured. Values are the means \pm standard error ( $\mathrm{n}=5$ ). * $\mathrm{P}<0.05$. (A) MIN6 group, MIN6 cells were exposed to $0.5 \%$ bovine serum albumin (BSA); (B) MIN6 + PA group, MIN6 cells were exposed to 0.5 mmol/1 palmitate plus 0.5\% BSA; (C) co-culture group, MIN6 cells were exposed to $0.5 \mathrm{mmol} / 1$ palmitate plus $0.5 \%$ BSA, and were co-cultured with untreated MS-1 cells; (D) co-culture + A group, MIN6 cells were exposed to $0.5 \mathrm{mmol} / 1$ palmitate plus $0.5 \% \mathrm{BSA}$, and were co-cultured with $\mathrm{MS}-1$ cells pre-treated with $10^{-6} \mathrm{~mol} / 1 \mathrm{Ang}(1-7)$; $(\mathrm{E})$ co-culture $+\mathrm{A}+7$ group, MIN6 cells were exposed to $0.5 \mathrm{mmol} / 1$ palmitate plus $0.5 \% \mathrm{BSA}$, and were co-cultured with $\mathrm{MS}-1$ cells pre-treated with $10^{-6} \mathrm{~mol} / 1 \mathrm{Ang}(1-7)$ and $10^{-6} \mathrm{~mol} / 1$ A779. Q1, Annexin V//PI+; Q2, Annexin V+/PI+ ${ }^{+}$3, Annexin $\mathrm{V}^{-} / \mathrm{PI}^{-}$; Q4, Annexin $\mathrm{V}^{+} / \mathrm{PI}^{-}$. 
ment around $\beta$ cells, and generates signals to maintain $\beta$ cells through islet microvascular endothelial cells in response to palmitate.

Islet microvascular endothelial cells secrete cytokines, such as hepatocyte growth factor (3), thrombospondin-1 (26), and laminins (27), which promote $\beta$ cell proliferation and inhibit differentiation through paracrine signaling pathways. Improvements in islet microvascular endothelial cell function may increase the levels of these cytokines, contributing to the positive effects of $A n g(1-7)$ on $\beta$ cell function. In future studies, it will be necessary to measure the levels of these cytokines, which was not done in the present study, to confirm their involvement in regulating $\beta$ cell function.

In conclusion, the results of the present study indicate that the ACE2/Ang(1-7)/Mas axis plays a permissive role in maintaining $\beta$ cell survival and function by improving islet microvascular endothelial cell function. Pharmacological and cell-based strategies aimed at increasing pancreatic ACE2/Ang(1-7)/Mas signaling activity may represent a novel approach for treating diabetes.

\section{Acknowledgements}

We thank Professor Hui Zhou (Tongji Hospital, Tongji Medical College, Huazhong University of Science and Technology, Hubei, China) for providing the MIN6 cells. This study was supported by a grant provided by the National Natural Science Foundation of China (no. 81070615).

\section{References}

1. Bearer EL and Orci L: Endothelial fenestral diaphragms: a quickfreeze, deep-etch study. J Cell Biol 100: 418-428, 1985.

2. Lammert E, Cleaver O and Melton D: Induction of pancreatic differentiation by signals from blood vessels. Science 294 564-567, 2001.

3. Johansson M, Mattsson G, Andersson A, et al: Islet endothelia cells and pancreatic beta-cell proliferation: studies in vitro and during pregnancy in adult rats. Endocrinology 147: 2315-2324, 2006.

4. Zhu W, Zhong C, Yu Y, et al: Acute effects of hyperglycaemia with and without exercise on endothelial function in healthy young men. Eur J Appl Physiol 99: 585-591, 2007.

5. Tikellis C, Wookey PJ, Candido R, et al: Improved islet morphology after blockade of the renin- angiotensin system in the ZDF rat. Diabetes 53: 989-997, 2004.

6. Yuan L, Li X, Li J, et al: Effects of renin-angiotensin system blockade on the islet morphology and function in rats with long-term high-fat diet. Acta Diabetol 50: 479-488, 2013.

7. Li X, Yuan L, Xu G, et al: Effect of renin angiotensin system blockade on the islet microvessel density of diabetic rats and its relationship with islet function. J Huazhong Univ Sci Technolog Med Sci 29: 684-688, 2009.

8. Donoghue M, Hsieh F, Baronas E, et al: A novel angiotensinconverting enzyme-related carboxypeptidase (ACE2) converts angiotensin I to angiotensin 1-9. Circ Res 87: E1-E9, 2000.
9. Danilczyk U and Penninger JM: Angiotensin-converting enzyme II in the heart and the kidney. Circ Res 98: 463-471, 2006.

10. Santos RA, Simoes e Silva AC, Maric C, et al: Angiotensin-(1-7) is an endogenous ligand for the $\mathrm{G}$ protein-coupled receptor Mas. Proc Natl Acad Sci USA 100: 8258-8263, 2003.

11. Benter IF, Yousif MH, Cojocel C, et al: Angiotensin-(1-7) prevents diabetes-induced cardiovascular dysfunction. Am J Physiol Heart Circ Physiol 292: H666-H672, 2007.

12. Shiota A, Yamamoto K, Ohishi M, et al: Loss of ACE2 accelerates time-dependent glomerular and tubulointerstitial damage in streptozotocin-induced diabetic mice. Hypertens Res 33: 298-307, 2010.

13. Patel VB, Bodiga S, Basu R, et al: Loss of angiotensin-converting enzyme-2 exacerbates diabetic cardiovascular complications and leads to systolic and vascular dysfunction: a critical role of the angiotensin II/AT1 receptor axis. Circ Res 110: 1322-1335, 2012.

14. Yuan L, Wang Y, Lu C and Li X: Angiotensin-converting enzyme 2 deficiency aggravates glucose intolerance via impairment of islet microvascular density in mice with high-fat diet. J Diabetes Res 2013: 405284, 2013.

15. Sampaio WO, Henrique de Castro C, Santos RA, et al: Angiotensin-(1-7) counterregulates angiotensin II signaling in human endothelial cells. Hypertension 50: 1093-1098, 2007.

16. Lovren F, Pan Y, Quan A, et al: Angiotensin converting enzyme-2 confers endothelial protection and attenuates atherosclerosis. Am J Physiol Heart Circ Physiol 295: H1377-H1384, 2008.

17. Bindom SM, Hans CP, Xia H, et al: Angiotensin I-converting enzyme type 2 (ACE2) gene therapy improves glycemic control in diabetic mice. Diabetes 59: 2540-2548, 2010.

18. Yuan L, Li X, Xu GL and Qi CJ: Effects of renin-angiotensin system blockade on islet function in diabetic rats. J Endocrinol Invest 33: 13-19, 2010

19. Oudit GY, Herzenberg AM, Kassiri Z, et al: Loss of angiotensinconverting enzyme- 2 leads to the late development of angiotensin II-dependent glomerulosclerosis. Am J Pathol 168: 1808-1820, 2006.

20. Yuan L, Li Y, Li G, et al: Ang(1-7) treatment attenuates $\beta$-cell dysfunction by improving pancreatic microcirculation in a rat model of type 2 diabetes. J Endocrinol Invest Dec 36: 931-937, 2013.

21. Bindom SM and Lazartigues E: The sweeter side of ACE2: physiological evidence for a role in diabetes. Mol Cell Endocrinol 302: 193-202, 2009.

22. Yan C, Kim D, Aizawa T, et al: Functional interplay between angiotensin II and nitric oxide: cyclic GMP as a key mediator. Arterioscler Thromb Vasc Biol 23: 26-36, 2003.

23. Burnier M and Zanchi A: Blockade of the renin-angiotensinaldosterone system: a key therapeutic strategy to reduce renal and cardiovascular events in patients with diabetes. J Hypertens 24: 11-25, 2006.

24. Song HJ, Xue WJ, Li Y, et al: Improved islet survival and funtion with rat endothelial cells in vitro co-culture. Transplant Proc 41: 4302-4306, 2009.

25. Duvillié B, Currie C, Chrones T, et al: Increased islet cell proliferation, decreased apoptosis, and greater vascularization leading to beta-cell hyperplasia in mutant mice lacking insulin. Endocrinology 143: 1530-1537, 2002.

26. Olerud J, Mokhtari D, Johansson M, et al: Thrombospondin-1: an islet endothelial cell signal of importance for $\beta$-cell function. Diabetes 60: 1946-1954, 2011.

27. Johansson A, Lau J, Sandberg M, et al: Endothelial cell signalling supports pancreatic beta cell function in the rat. Diabetologia 52: 2385-2394, 2009. 